\title{
INFINITE-ODS IN ARCWISE CONNECTED CONTINUA
}

\author{
ELDON J. VOUGHT
}

(Communicated by James E. West)

\begin{abstract}
The theorem is proved that a continuum that has only finitely many arc components (hence an arcwise connected continuum) and contains an $n$-od for every integer $n$ must contain an infinite-od.
\end{abstract}

FitzGerald [1, Corollary 4.8, p. 157] showed that a locally connected continuum that is an $n$-od for every positive integer $n$ is an $\infty$-od. Heath obtained the same conclusion by replacing locally connected with a hereditary decomposable condition (see Theorem A). In the same paper [2, Example 2, p. 477] she constructed a continuum that is an $n$-od for every positive integer $n$ but is not an $\infty$-od. Nall in [3, p. 245], generalizing a result of Sorgenfrey, gave sufficient conditions in order for a continuum to contain an $\infty$-od. In a conversation with the author, Nall and Hagopian raised the question of whether an arcwise connected continuum that contains an $n$-od for every $n$ must contain an $\infty$-od. The purpose of this paper is to give an affirmative answer to this question. In fact, the answer is yes under the weaker hypothesis that the continuum has only finitely many arc components. In the case of infinitely many arc components, however, the answer is no, and a continuum is constructed with an infinite number of arc components that contains an $n$-od for every $n$ but no $\infty$-od.

A continuum is a $\theta_{n}$-continuum if no subcontinuum separates it into more than $n$ components. A continuum $X$ is an $n$-od if there exists a subcontinuum $H$, called the $h u b$, such that $X \backslash H$ has at least $n$ components. If $X \backslash H$ has an infinite number of components then $X$ is an $\infty$-od. Note that a continuum $X$ is a $\theta_{n}$-continuum if and only if $X$ is not an $(n+1)$-od.

Example. There exists a continuum with an infinite number of arc components that contains an $n$-od for every $n$ but does not contain an $\infty$-od.

Let $X$ be a continuum irreducible between $a$ and $b$ that admits a monotone upper semicontinuous map $f$ onto $[0,1]$ such that $(\mathrm{i}) f^{-1}(0)=a$, $f^{-1}(1)=b$, (ii) $f^{-1}(y)$ is degenerate if $y \neq \frac{1}{n}$ for $n=3,4, \ldots$, (iii) $f^{-1}\left(\frac{1}{n}\right)$ is a simple $n$-od of diameter $\frac{1}{n}$ for $n=3,4, \ldots$, and (iv) $f^{-1}\left(\frac{1}{n}\right)$

Received by the editors September 3, 1991; presented to the Annual Spring Topology Conference at California State University, Sacramento on April 11, 1991.

1991 Mathematics Subject Classification. Primary 54F20; Secondary 54B15.

Key words and phrases. Infinite-od ( $\infty$-od), $n$-od, arc component, $\theta_{n}$-continuum, hereditarily decomposable, monotone upper semicontinuous decomposition, graph. 
has void interior for $n=3,4, \ldots$. The continuum $X$ has the aforementioned properties.

To proceed, we need the following two theorems.

Theorem A (Heath). If $W$ is a continuum and, for each $n$ there is a subcontinuum $K_{n}$ of $W$ such that (i) $W \backslash K_{n}$ has at least $n$ components and (ii) the closure of each component of $W \backslash K_{n}$. is hereditarily decomposable, then $W$ is an $\infty$-od [2, Theorem 2, p. 486].

Theorem B (Vought). If $W$ is a hereditarily decomposable, $\theta_{n}$-continuum, then $W$ admits a unique monotone, upper semicontinuous decomposition $\mathscr{D}$, the elements of which have void interior and for which $W / \mathscr{D}$ is a graph $[4$, Theorem 2, p. 635].

Lemma 1. Let $X$ be a continuum with only finitely many arc components and suppose $X$ contains no $\infty$-od. Then every subcontinuum of $X$ has only finitely many arc components and thus is decomposable. Hence $X$ is hereditarily decomposable.

Proof. Suppose $W$, a subcontinuum of $X$, has an infinite number of arc components. Since $X$ has only finitely many arc components, let $C_{1}, C_{2}, \ldots$ be a sequence of arc components of $W$ that lie in the same arc component $C$ of $X$. Because $C$ is arcwise connected, $C \backslash \bigcup_{i=1}^{\infty} C_{i} \neq \varnothing$. Choose $x$ in $C \backslash \bigcup_{i=1}^{\infty} C_{i}, w$ in $C_{1}$, and let $A_{1}=\langle w, x\rangle$ be an arc in $C$ from $w$ to $x$. Let $w^{\prime}=\operatorname{lub}_{A_{1}}\left\{y \mid y \in C_{1}\right\}$. Then $w^{\prime} \in C_{1}$. For, suppose not. There exists a sequence of points of $A_{1} \cap C_{1}, w_{1}, w_{2}, \ldots$, such that $w_{i}<w^{\prime}$ for $i=1,2, \ldots$ and $\lim w_{i}=w^{\prime}$. The subarc $\left\langle w_{1}, w^{\prime}\right\rangle$ of $A_{1}$ is not contained in $W$, so there is a subarc $\left\langle r_{1}, s_{1}\right\rangle$ of $\left\langle w_{1}, w^{\prime}\right\rangle$ such that $r_{1}, s_{1} \in W$ and $\left(r_{1}, s_{1}\right) \cap W=\varnothing$. Without loss of generality, assume that $w_{2} \geq s_{1}$. Then there exists a subarc $\left\langle r_{2}, s_{2}\right\rangle$ of the subarc $\left\langle w_{2}, w^{\prime}\right\rangle$ of $A_{1}$ such that $r_{2}, s_{2} \in W$ and $\left(r_{2}, s_{2}\right) \cap W=\varnothing$. Continuing, we obtain an infinite sequence $\left\langle r_{1}, s_{1}\right\rangle,\left\langle r_{2}, s_{2}\right\rangle, \ldots$ of subarcs of $A_{1}$ such that for $i=1,2, \ldots, r_{i}, s_{i} \in W$, $\left(r_{i}, s_{i}\right) \cap W=\varnothing$, and $\left(r_{i}, s_{i}\right) \cap\left(r_{j}, s_{j}\right)=\varnothing$ if $i \neq j$. Then $W \cup \bigcup_{i=1}^{\infty}\left\langle r_{i}, s_{i}\right\rangle$ is an $\infty$-od with hub $W$, a contradiction. So $w^{\prime} \in A_{1} \cap C_{1}$.

There exists a point $v>w^{\prime}$ in $A_{1}$ such that the arc $\left\langle w^{\prime}, v\right\rangle \cap W=\left\{w^{\prime}\right\}$. For, suppose not. There exists a sequence of points $v_{1}, v_{2}, \ldots$ in $A_{1}$ such that, for $i=1,2, \ldots, w^{\prime}<v_{i}, v_{i} \in W$ and $\lim v_{i}=w^{\prime}$. But $w^{\prime} \in C_{1}$ and $w^{\prime}=\operatorname{lub}_{A_{1}}\left\{y \mid y \in C_{1}\right\}$, so $\left\langle w^{\prime}, v_{i}\right\rangle \not \subset W$ for $i=1,2, \ldots$. Then by an analogous construction to that of the last paragraph, an $\infty$-od can be constructed that yields a contradiction. Let $K_{1}$ be a subarc of $\left\langle w^{\prime}, v\right\rangle$ with one end point $w^{\prime}$ such that $\operatorname{diam} K_{1} \leq 1$.

Repeat the above argument with the arc component $C_{2}$ and obtain an arc $K_{2}$ such that $K_{2}$ intersects $W$ only at an end point of $K_{2}$ and diam $K_{2} \leq \frac{1}{2}$. Clearly $K_{2}$ can be obtained such that $K_{1} \cap K_{2}=\varnothing$. Then in general, there is a sequence of mutually disjoint arcs $K_{1}, K_{2}, \ldots$ such that for each $i, i=$ $1,2, \ldots, K_{i}$ intersects $W$ only at an end point of $K_{i}$ and $\operatorname{diam} K_{i} \leq 1 / i$. Thus $W \cup \bigcup_{i=1}^{\infty} K_{i}$ is an $\infty$-od with hub $W$, a contradiction. This proves Lemma 1.

Lemma 2. Let $X$ be a continuum with only finitely many arc components and suppose $X$ contains no $\infty$-od. Then every subcontinuum of $X$ is a $\theta_{n}$-continuum for some $n$. 
Proof. Let $W$ be a subcontinuum of $X$ and suppose $W$ is an $n$-od for every $n$. Thus Theorem $\mathrm{A}(\mathrm{i})$ is true. By Lemma $1, W$ is hereditarily decomposable and so Theorem $\mathrm{A}$ (ii) is true. Hence $W$ is an $\infty$-od, a contradiction. Therefore, there exists an integer $n$ such that $W$ is a $\theta_{n}$-continuum and Lemma 2 is established.

Lemma 3. Let $X$ be a continuum with only finitely many arc components and suppose $X$ contains no $\infty$-od. Then every subcontinuum admits a unique monotone, upper semicontinuous decomposition, the elements of which have void interior and for which the quotient space is a graph. Furthermore, at most a finite number of the elements of the decomposition are nondegenerate.

Proof. Let $W$ be a subcontinuum of $X$. By Lemma $1, W$ is hereditarily decomposable. By Lemma $2, W$ is a $\theta_{n}$-continuum for some $n$. Thus, by Theorem B, $W$ admits the decomposition $\mathscr{D}$ of Lemma 3 for which $W / \mathscr{D}$ is a graph. By Lemma $1, W$ has only finitely many arc components. Since $W / \mathscr{D}$ is a graph, $\mathscr{D}$ contains only finitely many elements of order different from 2, where the order of an element of $\mathscr{D}$ is its order as an element of $W / \mathscr{D}$. There can be only finitely many nondegenerate elements of $\mathscr{D}$ of order 2; for otherwise, there would be infinitely many of these elements that are points on one edge of the graph $W / \mathscr{D}$, thus contradicting the fact that $W$ has only finitely many arc components.

Theorem (Main result). If $X$ is a continuum with only finitely many arc components and if $X$ contains an $n$-od for every $n$, then $X$ contains an $\infty$-od.

Proof. Suppose $X$ contains no $\infty$-od and let $X_{0}=X$. By Lemma 3, $X_{0}$ has a decomposition $\mathscr{D}_{0}$ such that $X_{0} / \mathscr{D}_{0}$ is a graph and at most a finite number of the elements of $\mathscr{D}_{0}$ are nondegenerate. Since $X_{0} / \mathscr{D}_{0}$ is a graph, there is a maximum integer $n_{0}$ such that $X_{0} / \mathscr{D}_{0}$ contains an $n_{0}$-od. But $X_{0}$ contains an $n$-od for every $n$, so it follows that an element $X_{1}$ of $\mathscr{D}_{0}$ has the property that $X_{1}$ contains an $n$-od for every $n$. By Lemma 3 again, $X_{1}$ has a decomposition $\mathscr{D}_{1}$ to a graph and at most a finite number of the elements of $\mathscr{D}_{1}$ are nondegenerate. Since $X_{1} / \mathscr{D}_{1}$ is a graph, there is a maximum integer $n_{1}$ such that $X_{1} / \mathscr{D}_{1}$ contains an $n_{1}$-od. But $X_{1}$ contains an $n$-od for every $n$, so it follows that an element $X_{2}$ of $\mathscr{D}_{1}$ has the property that $X_{2}$ contains an $n$-od for every $n$.

Continuing, we obtain continua $X_{0}, X_{1}, X_{2}, \ldots$ and decompositions $\mathscr{D}_{0}$, $\mathscr{D}_{1}, \mathscr{D}_{2}, \ldots$ such that for $k=1,2, \ldots, X_{k}$ is a nondegenerate element of the decomposition $\mathscr{D}_{k-1}$ of $X_{k-1}$ to a graph and $X_{k}$ contains an $n$-od for every $n$. Since $X_{k}$ is a nondegenerate element of $\mathscr{D}_{k-1}$ and $X_{k-1}$ has only finitely many arc components, for each $k, k=1,2, \ldots$, there is a half ray $H_{k-1}$ in $X_{k-1}$ that limits on a nondegenerate subcontinuum of $X_{k}$ and intersects no nondegenerate or vertex element of $\mathscr{D}_{k-1}$. The collection of these half rays is infinite but $X$ contains only finitely many arc components, so an infinite number of half rays lie in the same arc component of $X$. Without loss of generality, assume that for each $k, k=0,1, \ldots, H_{k}$ lies in the same arc component of $X$. Pick $x_{0}$ in $H_{0}$. Since $X_{k} / \mathscr{D}_{k}$ is a graph for each $k$, $k=1,2, \ldots$, there is a half ray $R_{k}$ in $H_{k}$ with end point $x_{k}$ and with the same limiting set as $H_{k}$ that intersects none of the at most finite number of arcs from $x_{0}$ to $X_{k}$. For each $k, k=1,2, \ldots$, let $A_{k}$ be an arc from $x_{0}$ to $x_{k}$ 
and let $C_{k}$ be an arc in $R_{k}$ such that $A_{k} \cap C_{k}=\left\{x_{k}\right\}$. Let $H=\overline{\bigcup_{i=1}^{\infty} A_{i}}$ and $K=H \cup\left(\overline{\bigcup_{i=1}^{\infty} C_{i}}\right)$. Observe that $\left(\overline{\bigcup_{i=1}^{\infty} A_{i}}\right) \backslash\left(\bigcup_{i=1}^{\infty} A_{i}\right)$ is a subset of $\bigcap_{i=1}^{\infty} X_{i}$. This follows from the fact that for every $n, X_{n} / \mathscr{D}_{n}$ is a graph, and so there are only a finite number of arcs from $x_{0}$ to $X_{n+1}$. Because the $X_{i}$ 's are nested, it is true that $\left(\overline{\bigcup_{i=1}^{\infty} C_{i}}\right) \backslash\left(\bigcup_{i=1}^{\infty} C_{i}\right)$ is also a subset of $\bigcap_{i=1}^{\infty} X_{i}$. Thus $K$ is an $\infty$-od with hub $H$. This is a contradiction and the theorem is established.

An immediate application of the theorem yields the following

Corollary. If $X$ is an arcwise connected continuum that contains an n-od for every $n$, then $X$ contains an $\infty$-od.

\section{REFERENCES}

1. R. W. FitzGerald, Connected sets with a finite disconnection property, Academic Press, New York, 1975, pp. 139-173.

2. Jo Heath, On n-ods, Houston J. Math. 9 (1983), 477-487.

3. Van C. Nall, On the presence of n-ods and infinite-ods, Houston J. Math. 15 (1989), 245247.

4. Eldon J. Vought, $\omega$-connected continua and Jones' $K$ function, Proc. Amer. Math. Soc. 91 (1984), 633-636.

Department of Mathematics and Statistics, California State University, Chico, CalIFORNIA 95929-0001

E-mail address: Vought@ecst.csuchico.edu 